\title{
Study of ribbon separation and magnetic reconnection rates
}

\author{
Wenbin Xie ${ }^{1,2}$, Hongqi Zhang ${ }^{2}$, Jun Lin ${ }^{3}$, and Haimin Wang ${ }^{4}$ \\ ${ }^{1}$ Department of Physics, Jilin Normal University, \\ Postbus 136000, Jilin,, China \\ email: wb_xie@bao.ac.cn \\ ${ }^{2}$ National Astronomical Observatories Chinese Academy of Sciences, \\ Postbus 100012 Beijing, China \\ email: hzhang@bao.ac.cn \\ ${ }^{3}$ Yunnan Astronomical Observatory, Chinese Academy of Sciences, \\ Postbus 650011, Yunnan, China \\ email: jlin@ynao.ac.cn \\ ${ }^{4}$ New Jersey Institute of Technology, \\ Postbus NJ07102, Newark \\ email: haimin@flare.njit.edu
}

\begin{abstract}
We study the correlation between the speed of two-ribbon separation and the magnetic flux density during the 2001 April 10 solar flare. A weak negative correlation is found between the ribbon separation speed $\left(V_{r}\right)$ and the longitudinal magnetic flux density $\left(B_{z}\right)$. In addition, we estimate the magnetic reconnection rate $\left(E_{r e c}\right)$. Along the flare ribbons, $E_{r e c}$ fluctuates in the small range except near the HXR source. The localized enhancement of the reconnection rate corresponds to the position of the HXR source.
\end{abstract}

Keywords. Solar activity, Solar flares, Solar magnetic fields

\section{Introduction}

Theoretically speaking, the separation speed of the ribbon expansion is different in the different background magnetic fields (Lin, 2004). Kitahara and Kurokawa (1990) suggested that the two flare ribbons separated much faster in the initial phase of ribbon expansion and then decelerated or even stopped when the $\mathrm{H} \alpha$ ribbon front invaded umbrae. Asai et al. (2004) found that the separating speed of $\mathrm{H} \alpha$ flare kernel decreased when the HXR burst occurred. Miklenic et al. (2007) found good temporal correlations between the time profiles of local and global reconnection rate, poynting flux and the HXR flux. Despite of the numerous studies in the past, the following question was not addressed: what is the quantitative relation between the ribbon separation speed and magnetic flux density in flares?

\section{Results}

On 2001 April 10 an X2.3 two-ribbon flare occurred in the NOAA AR $9415\left(S 22^{\circ}, W 01^{\circ}\right)$. The images of the flare ribbon are obtained from the TRACE. We use a magnetogram before the flare eruption obtained with the SOHO / MDI to measure photospheric magnetic flux density. To aid the tracking of the position of the flare ribbons, we choose 46 reference points along the ribbons and mark from $L_{0}$ to $L_{45}$. The locations of reference points L5, L6, L7 and L26, L27, L28, L29 correspond to the left and right HXR sources, respectively. 


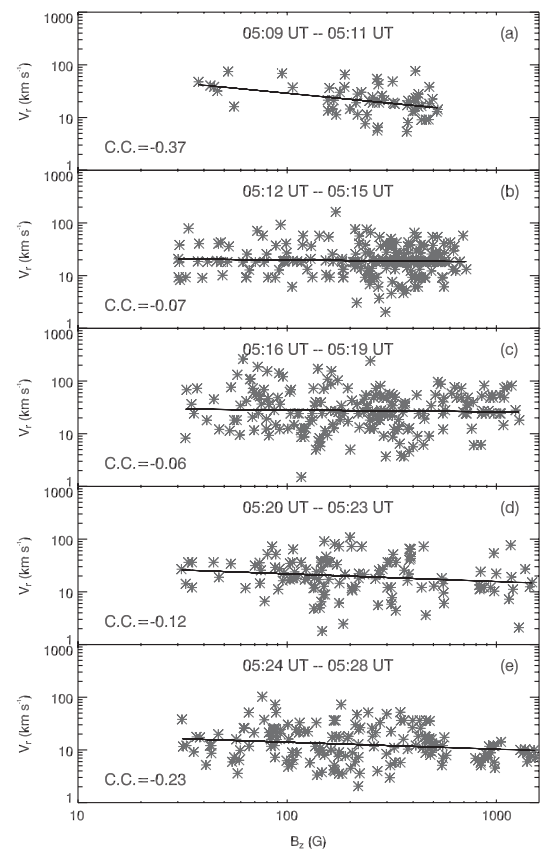

Figure 1. Statistical distribution of $V_{r} \mathrm{~s}$ and $B_{z} \mathrm{~s}$ in 5 time bins. The solid lines are the fits curves of $V_{r}$ and $B_{z}:(\mathrm{a}), V_{r}=168.5 \times B_{z}^{-0.38} ;(\mathrm{b})$, $V_{r}=23.8 \times B_{z}^{-0.04} ;(\mathrm{c}), V_{r}=32.4 \times B_{z}^{-0.03} ;(\mathrm{d})$, $V_{r}=40.8 \times B_{z}^{-0.15} ;(\mathrm{e}), V_{r}=26.4 \times B_{z}^{-0.13}$.
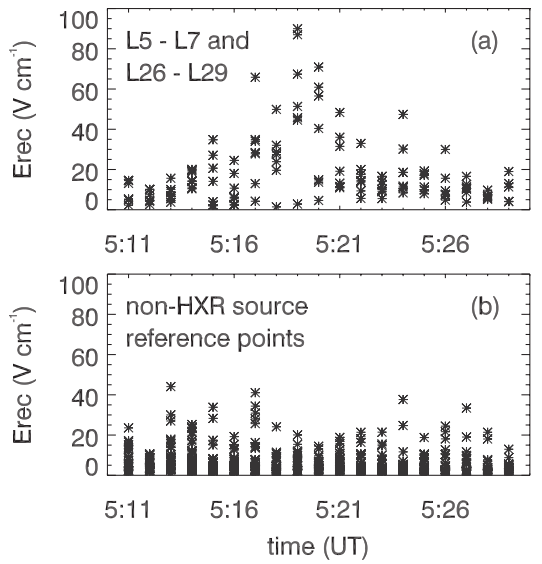

Figure 2. Distribution of $E_{\text {rec }}$ at the reference points in the left HXR source (L5-L7) and the right HXR source (L26-L29). (b): Distribution of $E_{r e c}$ at the other reference points.

(1) The separating speed of the flare ribbons is negatively correlated with the magnetic field. We analyzed the statistical distribution of the instantaneous speed of ribbon separation and the longitudinal magnetic flux density in each 4-minute time interval after 5:12 UT. Figure 1 shows the scatter plot of $V_{r} \mathrm{~s}$ as a function of the absolute value of $B_{z} \mathrm{~s}$ in five time bins. The maximum $V_{r}$ is about $260 \mathrm{~km} \mathrm{~s}^{-1}$ at a location corresponding to $B_{z} \mathrm{~s}<400 \mathrm{G}$; about $95 \mathrm{~km} \mathrm{~s}^{-1}$ when $B_{z} \mathrm{~s}$ are in the range from $400 \mathrm{G}$ to $1300 \mathrm{G}$, and about $30 \mathrm{~km} \mathrm{~s}^{-1}$ when $B_{z} \mathrm{~s}>1300 \mathrm{G}$. In Figure 1, the solid line is a fit to the data points in the form of (a) $V_{r}=168.5 \times B_{z}^{-0.38}$, (b) $V_{r}=23.8 \times B_{z}^{-0.04}$, (c) $V_{r}=32.4 \times B_{z}^{-0.03}$, (d) $V_{r}=40.8 \times B_{z}^{-0.15}$, (e) $V_{r}=26.4 \times B_{z}^{-0.13}$.

(2) Along the ribbons, $B_{z}$ and $V_{r}$ fluctuate significantly, and the $V_{r}$ is negatively correlated with the $B_{z}$, but $E_{r e c}$ fluctuates in the small range except near the HXR source. Figure 2 shows the Distribution of $E_{\text {rec }}$ of the reference points in the HXR source (Figure $2 \mathrm{a}$ ) and the non HXR source (Figure $2 \mathrm{~b}$ ). The localized enhancement of $E_{\text {rec }}$ corresponds to the position of HXR source, and the $E_{r e c} \mathrm{~s}$ are weak before and after the peak time of the flare in the position of HXR source.

\section{References}

Asai, A., Yokoyama, T., Shimojo, M., Masuda, S., Kurokawa, H., \& Shibata, K. 2004, ApJ, 611, 557

Kitahara, T. \& Kurokawa, H. 1990, SolPhys, 125, 321

Lin, J. 2004, SolPhys, 222, 115

Miklenic, C. H., Veronig, A. M., Vršnak, B., \& Hanslmeier, A. 2007, Astron. Astrophys., 461, 697 